\title{
Evaluation of Load Transmission to the Knee Joint in a Three- dimensional Femur Model Using a Finite Element Analysis Method
}

\author{
(D) Aybars Tekcan1, (D) Nusret Köse² \\ ${ }_{1}^{1}$ Medipol University Faculty of Medicine, Department of Orthopedics and Traumatology, istanbul, Turkey \\ 2Eskişehir Osmangazi University Faculty of Medicine, Department of Orthopedics and Traumatology, Eskişehir, Turkey
}

\section{Abstract}

Objective: This study aimed to evaluate and determine the effects of femoral anteversion (FAV) and femoral neck shaft angles (FNSA) changes on the medial and lateral compartments of the distal femur using finite element analysis (FEA).

Methods: The study was designed in two stages. First, the FEA was used to evaluate the imaginary environment (IE). Then, solid models were formed and tested in an experimental environment to validate the three-dimensional (3D) models. Three adult male cadaver femurs were scanned for the IE study. The computed tomography cross-sectional scans were reconstructed to provide a 3D surface model of cancellous and cortical bones. This model was accepted as a basic model, and this model was modified with software to create 42 models by using seven different FAV and six FNSA. These modified models were then analyzed to define mesh structure. The stress values were obtained after the FEA.

Results: In the lateral compartment of the distal femur (LCDF), the highest force recorded was $625.47 \mathrm{~N}$, and the lowest force recorded was $239.41 \mathrm{~N}$. In the medial compartment of the distal femur (MCDF), the highest force recorded was $910.59 \mathrm{~N}$ and the lowest force recorded was $524.53 \mathrm{~N}$. The standard femoral model (SFM), which had an FNSA of $135^{\circ}$ and FAV of $10^{\circ}$, was chosen due to the close resemblance of its anatomic features to human femur. According to SFM, a maximum decrease of $44 \%$ and an increase of $47 \%$ in LCDF and a maximum decrease of $28 \%$ and an increase of $26 \%$ in MCDF were observed.

Conclusion: In the study, we found that changed FAV and FNSA significantly affected LCDF compared with MCDF.

Keywords: Femoral anteversion, femur neck shaft angle, knee osteoarthritis, finite element analysis, biomechanics

\section{INTRODUCTION}

Knee alignment plays an important role in the formation and development of osteoarthritis (OA) $(1,2)$. The axis extending from the center of the femoral head to the intercondylar notch of the distal femur is called the mechanical axis of the femur. The axis extending from the center of the proximal tibia to the center of the ankle is called the mechanical axis of the tibia. The medial angle formed between the mechanical axis of the femur and the mechanical axis of the tibia is called the hip knee angle (HKA). The normal range of $\mathrm{HKA}$ is $0^{\circ}-2^{\circ}$ varus. Alternatively, the anatomical axis of the femur has an approximate $5^{\circ}-7^{\circ}$ of inclination difference compared with the mechanical axis. The lateral angle between the anatomic axes of the femur and tibia is called the femorotibial angle (FTA). The average FTA is approximately $178^{\circ}$ in men and $176^{\circ}$ in women (3). With daily activities, the knee is usually more loaded into the medial compartment. This loading difference may explain why medial $\mathrm{OA}$ is more common $(4,5)$.

The Framingham Osteoarthritis Study showed that the lateral and medial OA differed according to gender, and lateral $\mathrm{OA}$ was more common in women (6). The higher incidence of lateral $\mathrm{OA}$ in women can simply be explained by the high 
prevalence of valgus alignment in women $(7,8)$. Alternatively, Brouwer et al. (9) found that although varus alignment had a strong effect on $\mathrm{OA}$, valgus alignment had a limited effect on the development of OA. Sharma et al. (2) found an association between varus alignment and the increasing risk of radiological $\mathrm{OA}$, but they could not find this connection in valgus alignment. Furthermore, Lee et al. (10) showed that one-third of the knees with stage 4 lateral OA have a varus alignment on hip-kneeankle radiograph.

Lateral OA was previously explained by changes in the hip's morphology due to the limited effect of knee valgus alignment on lateral OA. In contrast with men, the hip adductor muscles in women are stronger than abductor muscles $(11,12)$. The reduction in the strength of abductor muscles decreases hip control and causes abnormal knee kinematics. The reduction in the femoral offset and presence of coxa valga decrease the abductor lever arm, which may cause an abductor moment reduction (13). In other words, reduction in the strength of hip abductor muscle may result in an increased valgus load in the knee $(14,15)$.

Weidow (16) studied the morphological changes of cartilage wear and found that more anterior wear occurs in medial knee $\mathrm{OA}$ and more posterior wear occurs in lateral knee OA. Weidow et al. (17) measured pelvic width, femoral offset, femoral neck length, and femoral neck shaft angles (FNSA) in patients with lateral knee OA. They emphasized that the anatomical variables in the pelvis and hip joints may change the distribution of the load on the knee joint and may have an effect on medial and lateral OA. In a gait analysis study, Weidow et al. (18) also found an association between the presence of lateral knee $\mathrm{OA}$ and the biomechanics of the hip joint. There is little information available in the literature on the relationship between the femoral anteversion (FAV) and FNSA and knee OA. Therefore, the purpose of this study is to evaluate and determine the effects of the changed FAV and FNSA regarding load transmission to the knee joint in 42 femur models using finite element analysis (FEA) method.

\section{METHODS}

This is a two-step study because it was not possible to study 42 different femoral models in vivo. First, FEA was used to evaluate the imaginary environment (IE). Solid models were then created and tested in an experimental environment (EE) to validate the three-dimensional (3D) models.

Three adult male cadaver femurs were scanned using Siemens Somatom Series, Sensation 16 Multi Detector (Forchheim, Germany) for IE study. Cancellous and cortical bone area between $2 \mathrm{~mm}$ and $4 \mathrm{~mm}$ and $5 \mathrm{~mm}$-to-9 $\mathrm{mm}$ intervals were obtained using cross-sectional (CT) scans, respectively. On the CT scan, the voxel dimension was 0.74 on $X$ and $Y$ coordinates and 0.7 on $Z$ coordinates. CT scans were reconstructed using 3D-Doctor (3.5.050106, Able Software, USA) software to provide a 3D surface model of cancellous and cortical bone (Figure 1). This model was accepted as a basic model and this basic model was modified with Autodesk AutoCAD 2005 (Autodesk, Inc., USA) software to create 42 models using seven different FAV $\left(-5^{\circ}, 0^{\circ}\right.$, $5^{\circ}, 10^{\circ}, 15^{\circ}, 20^{\circ}$, and $\left.25^{\circ}\right)$ and six different FNSA $\left(120^{\circ}, 125^{\circ}\right.$, $130^{\circ}, 135^{\circ}, 140^{\circ}$, and $145^{\circ}$ ) (Figure 1). These modified models were then analyzed with ANSYS Workbench 14.5 (ANSYS, Inc., USA) software to define the mesh structure (3D configuration like spider's web). The mesh structure is made up of elements and node units (Figure 2). The number of elements and nodes show a positive correlation with the complexity of the mesh structure. In our study, the mean numbers of nodes and elements were 74,000 and 44,000, respectively.

As a result, 21,846 nodes were intersected. A node cloud was formed with the intersected points using AutoCAD software to compare the stress value of different areas. A transactional scan was performed at $100 \mathrm{~mm}$ distal of the intertrochanteric region, and 15 regions were formed with a $15 \mathrm{~mm}$ distance between them. Stress distributions were evaluated in each region and in the lateral and medial femoral condyle. FEA was run and reaction forces on the fixation point and von Mises stresses on each node were recorded.

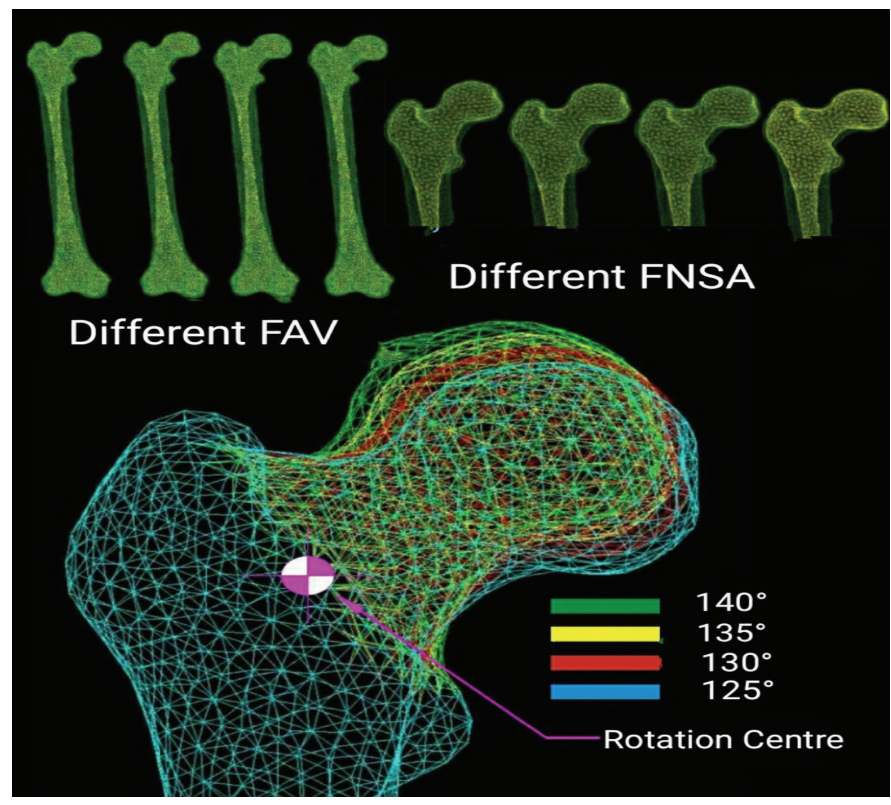

Figure 1. View of seven different FAV and six different FNSA created by software

FAV: Femoral anteversion, FNSA: Femoral neck shaft angles 
The stress value in the cortical bone elastic modulus is 14,217 $\mathrm{N} / \mathrm{mm}^{2}$ and Poisson's ratio is 0.32 , whereas the stress value in the cancellous bone elastic modulus is $1,000 \mathrm{~N} / \mathrm{mm}^{2}$ and Poisson's ratio is 0.3 . In this study, the bone tissue is treated as a homogeneous, isotropic, and linear material, even though it shows heterogeneous, non-linear and anisotropic features due to different structures of cortical and cancellous bone. This study aims to investigate the variables that affect the biomechanical properties and perform a comparative biomechanical analysis. Therefore, the average properties used are sufficiently accurate. A force of 1,150 $\mathrm{N}$ was applied vertically to the solid model during walking (Figure 3A). The solid models were fixed from the medial and lateral femoral condyle points (Figure 3B) (18).

As a result, 21,846 nodes were intersected. A node cloud was formed with the intersected points using AutoCAD software to compare the stress value of different areas. A transactional scan was performed at $100 \mathrm{~mm}$ distal of the intertrochanteric region, and 15 regions were formed with a $15 \mathrm{~mm}$ distance between them. Stress distributions were evaluated in each region and in the lateral and medial femoral condyle.

In a biomechanical real environmental study, the experimental bone model was created using cylinder industrial polyamide. Its mechanical properties have a close resemblance to the cortical bone tissue (19). A polyamide bone model was created

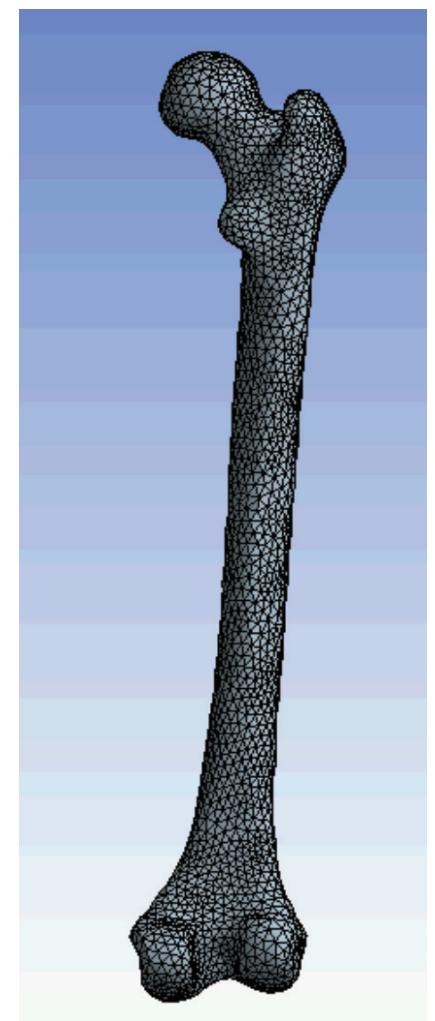

Figure 2. Mesh structure of model view by combining three components using two joints. The first and second joints were used to change the varus valgus and anteversion angles, respectively. Bars were used on joints to minimize the errors on varus valgus and anteversion angles (Figure 4). The sagittal view of $0^{\circ}, 10^{\circ}$, and $15^{\circ}$ angles and frontal view of $120^{\circ}, 125^{\circ}, 130^{\circ}, 135^{\circ}, 140^{\circ}$, and $145^{\circ}$ angles were selected to be used in biomechanical real environmental study, and the results were compared with the results of the imaginary studies. The polyamide model was fixed onto an aluminum platform using a cylindrical -shaped joint, which represented the lateral cortex allowing a rotation on the $\mathrm{X}$ axis. This platform was fixed onto a measurement frame using cylindrical beds to enable movement on the $\mathrm{Z}$ axis. A load cell was placed under the polyamide model in the same region, which represented the medial fixation points in the imaginary study. To prevent elasticity in the joints, a force of $500 \mathrm{~N}$ was selected as the load cell maximum measurement capacity. The force was applied vertically, and the steel balls were used to secure vertical force and enable moment forces (Figure 5). The results of the force applied to the joints were then transferred to the computer using a data logger.

Eskişehir Osmangazi University Faculty of Medicine Ethics Committee approved (date: 08.2.2007, project number: PR-0702-08-17).

\section{Statistical Analysis}

The statistical analysis was performed using the SPSS, version 20.0 for Windows (SPSS Inc., Chicago, IL, USA). The variables were investigated using Kolmogorov-Simirnov/Shapiro-Wilk's test to determine whether they are normally distributed. Mann-Whitney $\mathrm{U}$ test was used to compare the reaction forces

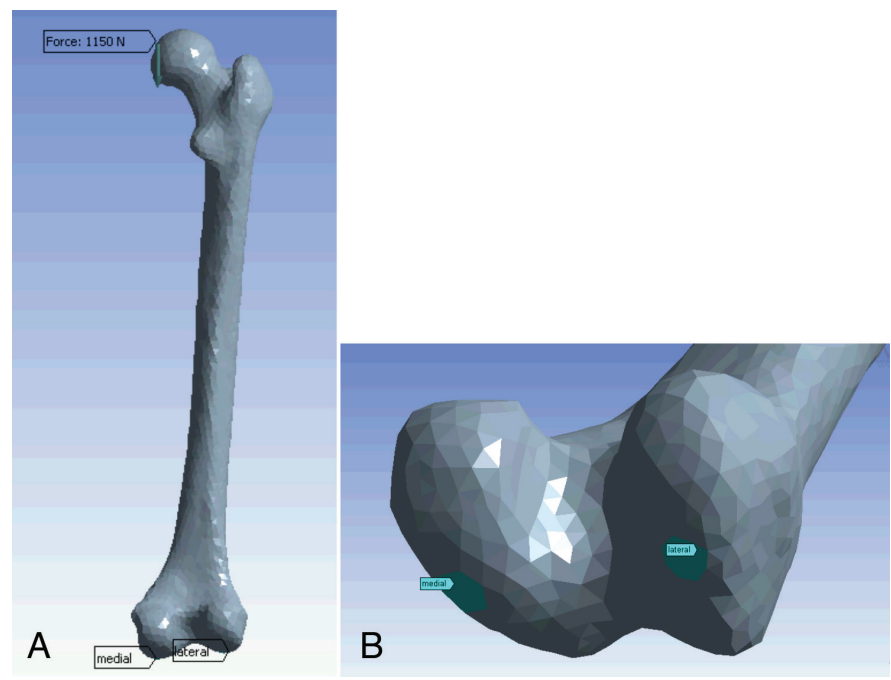

Figure 3. A) $1150 \mathrm{~N}$ load application point, B) medial and lateral fixation points 
between IE and EE. While investigating the association of the reaction forces between lateral compartment of the distal femur (LCDF) and medial compartment of the distal femur (MCDF), the correlation coefficients and their significance were calculated using Spearman's test. P value $<0.05$ was considered statistically significant.

\section{RESULTS}

The highest force was recorded on LCDF, in which FNSA and FAV were $145^{\circ}$ and $-5^{\circ}$, respectively. The lowest force was recorded on LCDF, in which FNSA and FAV were $120^{\circ}$ and $20^{\circ}$, respectively. The highest force was recorded on MCDF, in which FCSA and FAV were $120^{\circ}$ and 20 , respectively. The lowest force was recorded on MCDF, in which FCSA and FAV were $145^{\circ}$ and $-5^{\circ}$, respectively. Table 1 shows reaction force values in the MCDF and LCDF with

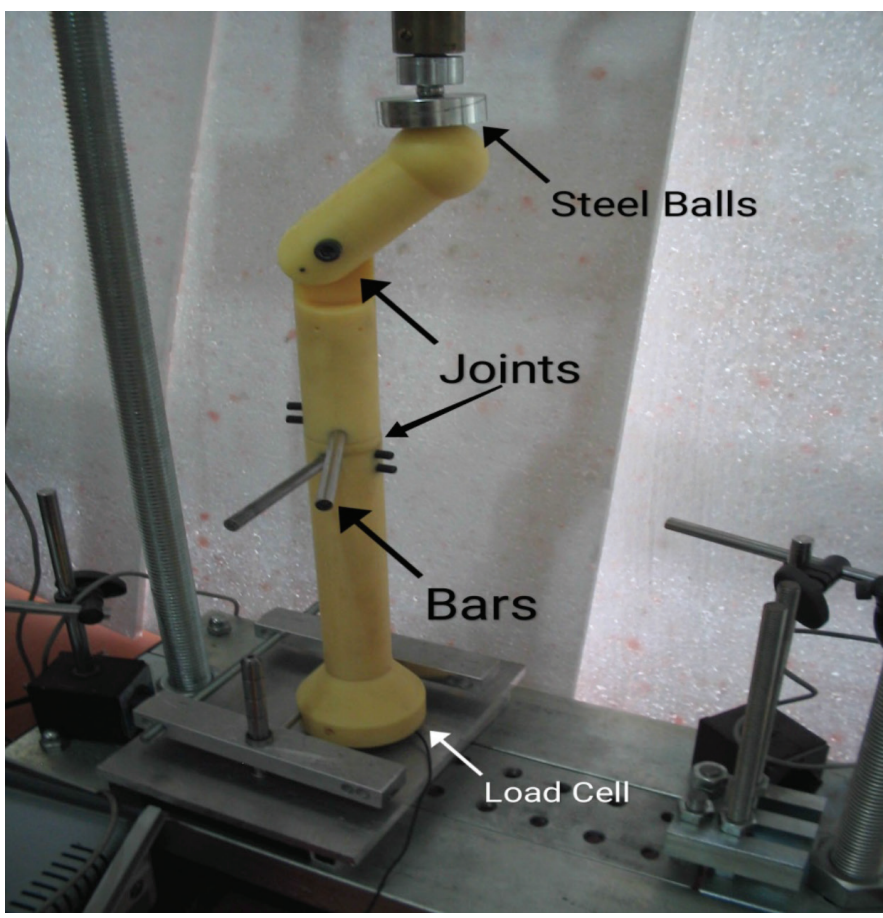

Figure 4. Polyamide femur model with bars and joints all FCNA ranging from $120^{\circ}$ to $145^{\circ}$ and all FAV from $-5^{\circ}$ to $25^{\circ}$ (Table 1).

A negative correlation was found between the lateral and medial parts of the distal femur after performing the correlation analysis of the reaction forces. That means when the load increases on one part of the knee, the load decreases on the other part and vice versa (Figure 6). A negative correlation was found between LCDF and FAV ( $r=-0.876 ; p=0.01)$, whereas a positive correlation was found between MCDF and FAV ( $r=896 ; p=0.006)$.

The standard femoral model (SFM), where FCSA was $135^{\circ}$ and FAV was $10^{\circ}$, was chosen due to the close resemblance of its anatomic features to the human femur. On SFM, the MCDF load was $424.75 \mathrm{~N}$, while the LCDF load was $725.25 \mathrm{~N}$. When FAV was $5^{\circ}$ and FNSA was $140^{\circ}$, the MCDF load was $638.94 \mathrm{~N} \mathrm{(a}$ decrease of 12\%) and the LCDF load was $511.06 \mathrm{~N}$ (an increase of 20\%). Table 2 shows the force change between MCDF and LCDF at different angles as a percentage compared with SFM (Table 2).

FCNA is kept constant at $135^{\circ}$. Only the change in FAV leads to a maximum of $10 \%$ change in MCDF compared with the standard femur, while a maximum change of $18 \%$ in LCDF is observed.

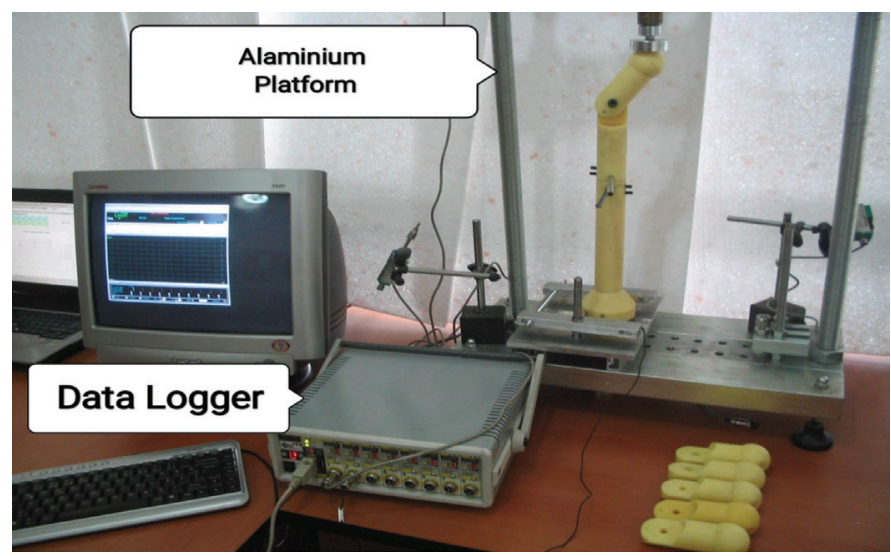

Figure 5. Aluminum platform and data logger

Table 1. Reaction force values of imaginary environment in the medial and lateral parts of the distal femur (N)

\begin{tabular}{|c|c|c|c|c|c|c|c|c|c|c|c|c|}
\hline & \multicolumn{2}{|l|}{$120^{\circ}$} & \multicolumn{2}{|l|}{$125^{\circ}$} & \multicolumn{2}{|l|}{$130^{\circ}$} & \multicolumn{2}{|l|}{$135^{\circ}$} & \multicolumn{2}{|l|}{$140^{\circ}$} & \multicolumn{2}{|l|}{$145^{\circ}$} \\
\hline & Med & Lat & Med & Lat & Med & Lat & Med & Lat & Med & Lat & Med & Lat \\
\hline$-5^{\circ}$ & 767.84 & 382.16 & 756.89 & 393.11 & 706.25 & 443.75 & 649.14 & 500.86 & 586.13 & 563.87 & 524.53 & 625.47 \\
\hline $0^{\circ}$ & 838.32 & 311.68 & 792.70 & 357.30 & 740.09 & 409.91 & 680.93 & 469.07 & 615.51 & 534.49 & 544.53 & 605.47 \\
\hline $5^{\circ}$ & 868.12 & 281.88 & 821.19 & 328.81 & 767.12 & 382.88 & 706.38 & 443.62 & 638.94 & 511.06 & 566.11 & 583.89 \\
\hline $10^{\circ}$ & 890.37 & 259.63 & 842.53 & 307.47 & 787.17 & 362.83 & 725.25 & 424.75 & 656.33 & 493.67 & 558.61 & 568.39 \\
\hline $15^{\circ}$ & 904.69 & 245.31 & 855.91 & 294.09 & 800.04 & 349.96 & 736.91 & 413.09 & 667.36 & 482.64 & 592.46 & 557.54 \\
\hline $20^{\circ}$ & 910.54 & 239.41 & 861.93 & 288.07 & 805.66 & 344.34 & 742.64 & 407.36 & 672.55 & 477.45 & 596.76 & 553.24 \\
\hline $25^{\circ}$ & 908.51 & 241.49 & 860.09 & 289.91 & 803.72 & 346.28 & 741.28 & 408.72 & 670.55 & 479.45 & 594.66 & 555.34 \\
\hline
\end{tabular}


In the $135^{\circ}$ model of FCNA, the effects of MCDF and LCDF at different FAV are shown in the table (Figure 7).

FAV is kept constant at $10^{\circ}$. Only the change in FCNA leads to a maximum of $23 \%$ change in MCDF compared with the standard femur, while a maximum change of $39 \%$ in LCDF is observed. In

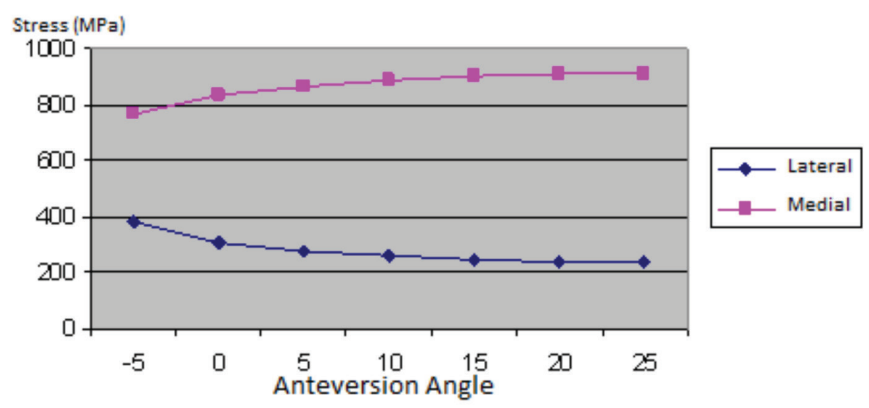

Figure 6. Negative correlation between the medial and lateral compartments

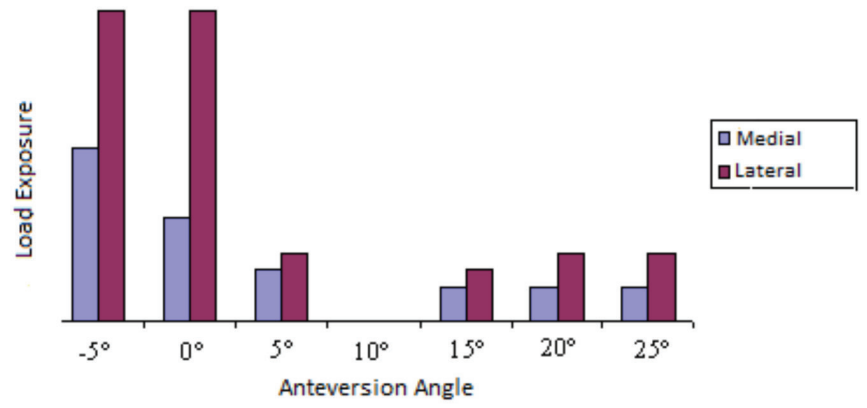

Figure 7. Medial and lateral effects when FNSA is fixed at $135^{\circ}$ and FAV is variable

FNSA: Femoral neck shaft angles, FAV: Femoral anteversion the $10^{\circ}$ model of FAV, the effects of MCDF and LCDF at different FCNA are shown in the table (Figure 8).

The values of biomechanical EE study are presented in Table 3. The reaction of forces gained from the real and virtual tests at $0^{\circ}, 10^{\circ}$, and $15^{\circ}$ anteversion angle, and $120^{\circ}, 125^{\circ}, 130^{\circ}, 135^{\circ}$, $140^{\circ}$, and $145^{\circ}$ shaft angle, were compared and contrasted using Mann-Whitney $U$ test. There are no statistical differences found between the tests conducted in EE and the tests conducted in IE $(p=0.12)$.

\section{DISCUSSION}

Many studies have supported that the tibiofemoral alignment is not only risk factor that affects compartment-specific knee OA. Malalignment of the lower leg, in either the varus or valgus direction, has influenced the distribution of load across the articular surfaces of the knee joint $(20,21)$. In valgus alignment,

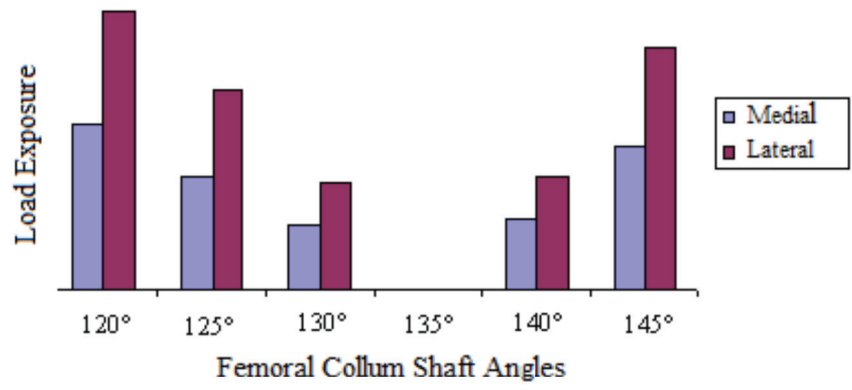

Figure 8. Medial and lateral effects when FAV is fixed at $10^{\circ}$ and FNSA is variable

FAV: Femoral anteversion, FNSA: Femoral neck shaft angles

Table 2. Comparison of standard femur model with other models using percentage

\begin{tabular}{|c|c|c|c|c|c|c|c|c|c|c|c|c|}
\hline & \multicolumn{2}{|l|}{$120^{\circ}$} & \multicolumn{2}{|l|}{$125^{\circ}$} & \multicolumn{2}{|l|}{$130^{\circ}$} & \multicolumn{2}{|l|}{$135^{\circ}$} & \multicolumn{2}{|l|}{$140^{\circ}$} & \multicolumn{2}{|l|}{$145^{\circ}$} \\
\hline & Med & Lat & Med & Lat & Med & Lat & Med & Lat & Med & Lat & Med & Lat \\
\hline$-5^{\circ}$ & $+\% 0.6$ & $-\% 10$ & $+\% 0.4$ & $-\% 0.7$ & $-\% 0.3$ & $+\% 0.4$ & $-\% 10$ & $+\% 18$ & $-\% 19$ & $+\% 33$ & $-\% 28$ & $+\% 47$ \\
\hline $5^{\circ}$ & $+\% 20$ & $-\% 34$ & $+\% 13$ & $-\% 23$ & $+\% 0.6$ & $-\% 10$ & $-\% 0.3$ & $+\% 0.4$ & $-\% 12$ & $+\% 20$ & $-\% 22$ & $+\% 37$ \\
\hline $10^{\circ}$ & $+\% 23$ & $-\% 39$ & $+\% 16$ & $-\% 28$ & $+\% 0.9$ & -\%15 & SFM & SFM & $-\% 10$ & $+\% 16$ & $-\% 20$ & $+\% 34$ \\
\hline $20^{\circ}$ & $+\% 26$ & $-\% 44$ & $+\% 19$ & $-\% 32$ & $+\% 11$ & $-\% 19$ & $+\% 0.2$ & $-\% 0.4$ & $-\% 0.7$ & $+\% 12$ & $-\% 18$ & $+\% 30$ \\
\hline $25^{\circ}$ & $+\% 25$ & $-\% 43$ & $+\% 19$ & $-\% 32$ & $-\% 11$ & $-\% 18$ & $+\% 0.2$ & $-\% 0.4$ & $-\% 0.8$ & $+\% 13$ & $-\% 18$ & $+\% 31$ \\
\hline
\end{tabular}

Table 3. Reaction force values of experimental environment $(\mathrm{N})$

\begin{tabular}{|l|l|l|l|l|l|l|}
\hline & $120^{\circ}$ medial & $125^{\circ}$ medial & $130^{\circ}$ medial & $135^{\circ}$ medial & $140^{\circ}$ medial & $145^{\circ}$ medial \\
\hline $0^{\circ}$ & 363.7 & 327.5 & 323.3 & 277.0 & 255.8 & 221.1 \\
\hline $10^{\circ}$ & 376.0 & 354.2 & 335.0 & 314.2 & 264.2 & 239.3 \\
\hline $25^{\circ}$ & 379.7 & 360.0 & 352.1 & 320.5 & 288.0 & 250.1 \\
\hline
\end{tabular}


the medial compartment continues to bear the load until excessive valgus occurs $(22,23)$. Therefore, only valgus alignment affected the development and progression of $\mathrm{OA}$ in the knee (9). Alternatively, Felson et al. (24) found that valgus alignment increased the risk of knee OA and lateral cartilage damage.

There are studies supporting the association of hip and pelvic geometry with compartment-specific knee OA. Weidow et al. (17) evaluated the pelvic width, femoral offset, and femoral neck length in patients with lateral and medial knee OA. In the same study, they evaluated these features in a normal hip group and hip OA group separately. In the group without hip OA, lateral OA was associated with a wider pelvis and shorter femoral neck and femoral offset. They found a coexistence of hip OA with lateral knee $O A$ and low incidence of hip changes in those with medial knee OA. Patients with lateral knee OA had increased FNSA. Their findings suggested that the occurrence of lateral and medial OA had a biomechanical background originating from the pelvis and hip anatomy (17). They also found an association between the presence of lateral knee OA and the biomechanics of the hip joint in gait analysis (18).

Boissonneault et al. (25) evaluated the association between tibiofemoral alignment, FNSA, femoral neck length, femoral offset, height of hip center, and abductor lever arm and compartment-specific knee OA. Lateral compartment OA was associated with increased abductor angle, increased FNSA (more valgus), and reduced femoral offset. Medial compartment OA was associated with reduced abductor angle and decreased FNSA (more varus) (25). In these studies, the anteversion was not evaluated.

In our study, we examined the relationship between FAV and FNSA regarding load transmission to the knee joint. Forty-two different femur models with six different FAV and seven different FNSA were investigated. The load on MCDF increased when FAV was decreased. The load on LCDF increased when FAV was increased. There is an increased load on MCDF when FNSA is decreased (more varus), and there is an increased pressure on LCDF when FNSA is increased (more valgus). FNSA was fixed at $135^{\circ}$, and FAV is reduced by $-5^{\circ}$. The distribution of the load was down to $61 \%$ on the medial part of the knee and $39 \%$ on the lateral part. The equal distribution of the load on both parts of the knee is achieved at $140^{\circ}$ and $145^{\circ}$ including all the anteversion angles.

In SFM, $63 \%$ of forces transmitted to the medial part of the knee and $37 \%$ to lateral part. In the study, the forces transmitted disproportionally from the hip to the knee joint. FNSA is kept constant at $135^{\circ}$. Only the change in FAV leads to a maximum of
$10 \%$ change in MCDF compared with the standard femur, while a maximum change of $18 \%$ in LCDF is observed. FAV is kept constant at $10^{\circ}$. Only the change in FNSA leads to a maximum of $23 \%$ change in MCDF compared with the standard femur, while a maximum change of $39 \%$ in LCDF is observed.

Table 2 is a different expression of the load change shown in Table 1 and provides a better understanding. When the increase or decrease in loads is considered to affect the lateral or medial side, the effect on the LCDF of the index is greater than MCDF. On examining the table carefully, LCDF numeric values are higher than those of MCDF.

\section{Study Limitations}

Our study has some limitations. First, when addressing FE models of bones, two key components, the geometry and material parameters, are essential. Both can be estimated from CT data but require a lot of approximation. Second, FE model did not consider the known local anisotropic behavior of the bone tissue. Third, even though inhomogeneus Young's modulus was represented different $E(p)$ relations in the cortical and trabecular subregions by Yosibash et al. (26), in our study the bone tissue was accepted as a homegeneous, izotrophic and lineer material. It should be noted that there were no statistical differences found between the test conducted in the EE and the test conducted in IE $(p=12)$. This statistical analysis showed that the EE study validated IE. Accurate methods for predicting and monitoring in vivo bone strength are of major importance in clinical applications. FEA is becoming a commonly used tool for the numerical analysis of the biomechanical response of human bones. Fourth, in this study, we need to mimic the joint contact force that is applied on the head of the femur during the complete gait cycle for the activities. The orientation and application of hip contact force are important because the dominant effect in the normal walking is hip contact force. A force of 2,460 N was expected to apply to the femoral head with an angle of $23^{\circ}$ at the frontal plane, $6^{\circ}$ at the sagittal plane, and a force of $1,700 \mathrm{~N}$ was expected with an angle of $24^{\circ}$ at the frontal plane and $15^{\circ}$ at the sagittal plane, related to the pull of abductor muscles at the trochanter major in the stance phase of the walking cycle. However, during the solid model experiments, the force applied to the femoral head caused the rotation of the model in the frame of measurement, and the optimum conditions cannot be obtained. We followed Peña et al. (27) and Sathasivam and Walker (19) studies, and a force of $1150 \mathrm{~N}$ was applied vertically to the femoral head to comply with the forces at the upper end of the femur. 
Wright et al. (28) found a correlation between the proximal and distal femoral geometry. The medial trochlear inclination angle correlated with FNSA and mediolateral (ML) femoral offset. The absence of ML femoral offset and the distal femur morphology are the shortcomings of our study (28).

Changing FNSA, which is essential in hip biomechanics, affects the medial and lateral distributions of the force applied to the knee joint. In the alterations of FNSA and FAV, not only does the distribution of force on hip join change, but also the knee joint is affected. Coskun Benlidayi et al. (29) found that people with FNSA above $134.4^{\circ}$ have an eightfold increased risk of developing severe knee OA.

Brouwer et al. (9) and Sharma et al. (21) found that valgus alignment affects the development and progression of knee OA. However, Felson et al. (24) found that valgus alignment increased the risk of knee OA. Neglecting hip and pelvic biomechanics while studying the effects of tibiofemoral alignment on compartmentspecific knee OA may be the cause of this conflict.

\section{CONCLUSION}

This biomechanical study filled the gap in the literature by evaluating the load distribution on the distal femur by the hip anteversion effect. In the study, we found that the changed biomechanics of the hip had a significant effect on the knee joint, and LCDF was affected more than MCDF. Determining the forces that affect the knee during different FNSA and FAV help understand the difference between the lateral and medial knee $\mathrm{OA}$. It is difficult to say that only the geometry of hip and pelvis is responsible for developing lateral knee OA. The geometry of hip and pelvis and valgus alignment may contribute to the development of lateral OA. Further dynamic studies, which evaluate hip and knee biomechanics together, are needed to better explain the occurrence of lateral OA.

\section{Ethics}

Ethics Committee Approval: Eskişehir Osmangazi University Faculty of Medicine Ethics Committee approved (date: 08.2.2007, project number: PR-07-02-08-17).

Informed Consent: Human studies were not conducted in this study therefore patient consent was not required.

Peer-review: Externally and internally peer-reviewed.

\section{Authorship Contributions}

Concept: N.K., Design: N.K., A.T., Data Collection or Processing: A.T., Analysis or Interpretation: A.T., Literature Search: A.T., Writing: A.T.
Conflict of Interest: No conflict of interest was declared by the authors.

Financial Disclosure: The authors declared that this study received no financial support.

\section{REFERENCES}

1. Sharma L, Chmiel JS, Almagor O, Felson D, Guermazi A, Roemer F, et al. The role of varus and valgus alignment in the initial development of knee cartilage damage by MRI: the MOST study. Ann Rheum Dis 2013;72:235-40.

2. Sharma L, Song J, Dunlop D, Felson D, Lewis CE, Segal N, et al. Varus and valgus alignment and incident and progressive knee osteoarthritis. Ann Rheum Dis 2010;69:1940-5.

3. Luo CF. Reference axes for reconstruction of the knee. Knee 2004;11:251-7.

4. Andriacchi TP. Dynamics of knee malalignment. Orthop Clin North Am 1994;25:395-403.

5. Schipplein OD, Andriacchi TP. Interaction between active and passive knee stabilizers during level walking. J Orthop Res 1991;9:113-9.

6. Felson DT, Nevitt MC, Zhang Y, Aliabadi P, Baumer B, Gale D, et al. High prevalence of lateral knee osteoarthritis in Beijing Chinese compared with Framingham Caucasian subjects. Arthritis Rheum 2002;46:121722.

7. Zhai G, Ding C, Cicuttini F, Jones G. A longitudinal study of the association between knee alignment and change in cartilage volume and chondral defects in a largely non-osteoarthritic population. J Rheumatol 2007:34:181-6.

8. Wise BL, Niu J, Yang M, Lane NE, Harvey W, Felson DT, et al. Patterns of compartment involvement in tibiofemoral osteoarthritis in men and women and in whites and African Americans. Arthritis Care Res (Hoboken) 2012;64:847-52.

9. Brouwer GM, van Tol AW, Bergink AP, Belo JN, Bernsen RM, Reijman $M$, et al. Association between valgus and varus alignment and the development and progression of radiographic osteoarthritis of the knee. Arthritis Rheum 2007;56:1204-11.

10. Lee SC, Gondalia V, Hwang BY, Ahn HS, Lee CK, Hunter DJ, et al. Is endstage lateral osteoarthritic knee always valgus? Mechanical alignment analysis and radiographic severity assessment. J Orthop Traumatol 2016;17:35-40

11. Mendiguchia J, Ford KR, Quatman CE, Alentorn-Geli E, Hewett TE. Sex differences in proximal control of the knee joint. Sports Med 2011;41:541-57.

12. Willson JD, Ireland ML, Davis I. Core strength and lower extremity alignment during single leg squats. Med Sci Sports Exerc 2006;38:945-52.

13. Callaghan J, Rosenberg A, Rubash H. The adult hip. 2nd edn. Philadelphia, PA: Lippincott Williams \& Wilkins; 2007.p.87-8.

14. Howard JS, Fazio MA, Mattacola CG, Uhl TL, Jacobs CA. Structure, sex, and strength and knee and hip kinematics during landing. J Athl Train 2011;46:376-85.

15. Jacobs CA, Uhl TL, Mattacola CG, Shapiro R, Rayens WS. Hip abductor function and lower extremity landing kinematics: sex differences. J Athl Train 2007:42:76-83.

16. Weidow J. Lateral osteoarthritis of the knee. Etiology based on morphological, anatomical, kinematic and kinetic observations. Acta Orthop Suppl 2006;77:3-44. 
17. Weidow J, Mars I, Kärrholm J. Medial and lateral osteoarthritis of the knee is related to variations of hip and pelvic anatomy. Osteoarthritis Cartilage 2005;13:471-7.

18. Weidow J, Tranberg R, Saari T, Kärrholm J. Hip and knee joint rotations differ between patients with medial and lateral knee osteoarthritis: gait analysis of 30 patients and 15 controls. J Orthop Res 2006;24:1890-9.

19. Sathasivam S, Walker PS. A computer model with surface friction for the prediction of total knee kinematics. J Biomech 1997;30:177-84.

20. Jacobs CR. Numerical simulation of bone adaptation to mechanical loading. Dissertation for the degree of doctor of Philosophy. Stanford University. California, PhD Thesis. 1994

21. Sharma L, Song J, Felson DT, Cahue S, Shamiyeh E, Dunlop DD. The role of knee alignment in disease progression and functional decline in knee osteoarthritis. JAMA 2001;286:188-95.

22. Tetsworth K, Paley D. Malalingment and degenerative arthropaty. Orthop Clin North Am 1994;25:367-77.

23. Johnson F, Leitl S, Waugh W. The distribution of load across the knee. A comparison of static and dynamic measurements. J Bone Joint Surg Br 1980;62:346-9.

24. Felson DT, Niu J, Gross KD, Englund M, Sharma L, Cooke TD, et al. Valgus malalignment is a risk factor for lateral knee osteoarthritis incidence and progression: findings from the Multicenter Osteoarthritis Study and the Osteoarthritis Initiative. Arthritis Rheum 2013;65:355-62.
25. Boissonneault A, Lynch JA, Wise BL, Segal NA, Gross KD, Murray DW, et al. Association of hip and pelvic geometry with tibiofemoral osteoarthritis: multicenter osteoarthritis study (MOST). Osteoarthritis Cartilage 2014;22:1129-35.

26. Yosibash Z, Trabelsi N, Milgrom C. Reliable simulations of the human proximal femur by high-order finite element analysis validated by experimental observations. J Biomech 2007;40:3688-99.

27. Peña E, Calvo B, Martínez MA, Palanca D, Doblaré M. Finite element analysis of the effect of meniscal tears and meniscectomies on human knee biomechanics. Clin Biomech (Bristol, Avon) 2005;20:498-507.

28. Wright SJ, Boymans TA, Grimm B, Miles AW, Kessler O. Strong correlation between the morphology of the proximal femur and the geometry of the distal femoral trochlea. Knee Surg Sports Traumatol Arthrosc 2014;22:2900-10.

29. Coskun Benlidayi I, Guzel R, Basaran S, Aksungur EH, Seydaoglu G. Is coxa valga a predictor for the severity of knee osteoarthritis? A crosssectional study. Surg Radiol Anat 2015;37:369-76. 